\title{
Influence du climat tropical humide et du niveau alimentaire sur la thermorégulation et les performances de croissance et d'abattage de jeunes boucs créoles en Guadeloupe
}

\author{
D. SERGENT ${ }^{(*)}$, P. BERBIGIER, S.A. SOPHIE \\ INRA - Unité de Bioclimatologie animale \\ Centre de Recherches des Antilles et de la Guyane \\ Domaine de Gardel - F 97160 Le Moule, Guadeloupe
}

\begin{abstract}
Résumé
Vingt-quatre jeunes boucs créoles de Guadeloupe (Antilles françaises) âgés de $246 \pm 45 \mathrm{j}$ et pesant $12,0 \pm 1,5 \mathrm{~kg}$ à la mise en cage, sont soumis à deux conditions climatiques (soleil $\mathbf{S}$ ou ombre $\mathrm{O}$ ) et à deux niveaux d'apport alimentaire (niveaux de concentré haut $\mathbf{H}$ ou bas $\mathbf{B}$ ) selon un schéma factoriel $2 \times 2$. Les premiers sont exposés en plein soleil alors que les seconds sont placés sous un toit de tôle ondulée. Quotidiennement, les animaux reçoivent à volonté du Pangola : les boucs des lots $\mathrm{H}$ reçoivent en plus $270 \mathrm{~g}$ de matière sèche de concentré, ceux des lots $\mathrm{B} 90 \mathrm{~g}$. L'expérience dure 10 mois.
\end{abstract}

La température rectale (TR) des boucs $B$ est supérieure à celle des boucs $H$ quand il fait le plus chaud alors que le rythme respiratoire (RR) reste indépendant du niveau alimentaire. Le niveau d'ingestion des boucs $\mathrm{H}$ est supérieur à celui des boucs $\mathrm{B}(617 \mathrm{~g} \mathrm{MS} / \mathrm{j}$ is $516 \mathrm{~g} \mathrm{MS} / \mathrm{j}$, $P<0,001)$. La vitesse de croissance $(54,5 \mathrm{~g} / \mathrm{j}$ vs $44,2 \mathrm{~g} / \mathrm{j})$, le poids vif vide $(23,4 \mathrm{~kg}$ vs $20,1 \mathrm{~kg})$, le poids de carcasse $(13,7 \mathrm{~kg}$ vs $11,6 \mathrm{~kg})$ sont toujours significativement plus élevés $(\mathrm{P}<0,001)$ chez les animaux $\mathrm{H}$. Le rendement vrai de carcasse n'est pas affecté.

L'exposition au soleil entraine un RR et une TR plus élevés qu'à l'ombre. Elle augmente la quantité de matière sèche ingérée $(587 \mathrm{~g} / \mathrm{j}$ vs $545 \mathrm{~g} / \mathrm{j}, \mathrm{P}<0,05)$, le poids de carcasse $(13,0 \mathrm{~kg}$ vs $12,2 \mathrm{~kg}, \mathrm{P}<0,05)$, le poids du foie $(1,65 \%$ du poids vif vide $v s 1,41 \%, \mathrm{P}<0,001)$ et diminue le pourcentage de gras interne $(4,9 \%$ vs $6,4 \%, \mathrm{P}<0,05)$.

Les boucs créoles tolèrent bien les conditions quasi naturelles les plus chaudes que l'on puisse trouver en Guadeloupe et leur élevage en plein air intégral, sous réserve d'une disponibilité alimentaire et d'un état sanitaire satisfaisants, n'est pas un facteur limitant puisque le bilan zootechnique est positif.

Mots clés : Tropiques humides, Antilles françaises, jeunes boucs créoles, régulation thermique, production de viande.

\section{Introduction}

Les performances des caprins dans les pays tropicaux sont dépendantes notamment des conditions climatiques et des disponibilités alimentaires. La Guadeloupe, archipel de l'Océan Atlantique au climat tropical humide à saison sèche marquée, possède une

(*) Adresse actuelle : INRA, Station de Bioclimatologie, 78850 Thiverval-Grignon (France). 
population importante de caprins créoles (40000 têtes) qui sont exploités essentiellement pour la viande. Certaines caractéristiques physiologiques et zootechniques des caprins créoles ont été inventoriées (AleXandre 1983, Chemineau et al., 1984, Sergent 1985, Sergent et al., 1985). Pour compléter ces travaux, la présente étude a pour objectif d'évaluer leur aptitude à la production de viande et d'analyser les effets des conditions climatiques et du niveau alimentaire. Pour cela, de jeunes boucs créoles ont été soumis à deux situations climatiques extrêmes (plein soleil avec averses tropicales ou abri) et à deux niveaux d'apport alimentaire afin d'induire une différence de thermogénèse (production de chaleur).

\section{Matériel et méthodes}

L'expérience dure 10 mois (septembre 1982- juin 1983) et se situe dans la partie nord-est (Grande-terre) de la Guadeloupe $\left(16^{\circ} \mathrm{N}, 61^{\circ} \mathrm{O}\right)$, sur le Domaine INRA de Gardel, dont la pluviométrie annuelle est de $1200 \mathrm{~mm}$ et la température moyenne annuelle de $25^{\circ} \mathrm{C}$. Vingt-quatre jeunes boucs créoles, sevrés à $8 \mathrm{~kg}$, pesant $12,0 \pm 1,5$ $\mathrm{kg}$ et âgés de $246 \pm 45 \mathrm{j}$ sont, en juillet 1982, répartis en 6 blocs en fonction du poids et du gain de poids moyen quotidien (G.M.Q.) des 70 jours précédents. Un animal de chaque bloc est ensuite affecté au hasard à un des quatre traitements suivants :

- Traitement 1 : animaux exposés àu soleil, nourris à un haut niveau d'alimentation (SH).

- Traitement 2 : animaux au soleil, et à bas niveau d'alimentation (SB).

- Traitement 3: animaux à l'ombre, et à haut niveau d'alimentation $(\mathrm{OH})$.

- Traitement 4 : animaux à l'ombre, et à bas niveau d'alimentation (OB).

L'ensemble constitue donc un dispositif factoriel $2 \times 2$, avec un effet "bloc » et deux variables : soleil-ombre (S-O) et niveau haut-bas (H-B). Bien que les traitements ne soient pas équilibrés sur les paramètres température rectale et rythme respiratoire, il n'y a pas de différence significative sur ces deux facteurs.

En août 1982, chaque animal est placé, sans attache, dans une cage individuelle de $1,50 \mathrm{~m}$ sur $0,60 \mathrm{~m}$ à caillebotis en bois. Les cages des traitements « soleil » sont placées en plein air sur une aire bétonnée, l'auge face à l'est (direction des vents alizés), et des contre-vents protègent la nourriture de la pluie ; celles des traitements " ombre » sont placées sous un abri en tôle fermé sur les côtés est et sud (protection contre la pluie).

Les boucs sont alimentés à volonté de Pangola en vert (Digitaria decumbens) fraîchement coupé à un âge de repousse d'environ $40 \mathrm{j}$. Sa concentration en énergie et en matières azotées digestibles estimé à partir de l'analyse chimique (INRA, 1978) et confirmé par deux expériences de digestibilité effectuées sur des boucs Créoles en croissance (SERGENT, XANDE, Berbigier, données non publiées) est présenté dans le tableau $1 \mathrm{a}$. Les animaux disposent d'eau à volonté. A cette ration sont ajoutés $300 \mathrm{~g}$ par jour d'aliment concentré composé (270 g MS) pour constituer le haut niveau alimentaire et $100 \mathrm{~g}$ (90 g MS) pour le bas niveau. Cet aliment (Cabri-Herbage, Grands-Moulins des Antilles) se compose de son de blé (65\% MS), de blé (20\% MS), de tourteau de soja $(10,6 \% \mathrm{MS})$, d'un prémix $(3,8 \% \mathrm{MS})$ et de maïs $(0,6 \% \mathrm{MS})$. Sa valeur nutritive est présentée dans le tableau 1 a.

Chez les boucs créoles, la digestibilité et donc la teneur en énergie métabolisable (EM) de la ration n'est pas diminuée, malgré l'association fourrage-concentré (XANDE, 
communication personnelle). La digestibilité de l'énergie (mesurée par analyse chimique, DIV) et la teneur en matières azotées totales (MAT) du Pangola varient (tabl. $1 \mathrm{~b})$; les moyennes de l'ensemble des données sont respectivement Dì $=66,6 \pm 5,8$ $(\mathrm{n}=39)$ et $\mathrm{MAT}=126 \pm 27 \mathrm{~g} / \mathrm{kg}$ MS $(\mathrm{n}=30)$. Toutefois, les valeurs théoriques correspondent bien à la moyenne des mesures expérimentales et nous pouvons donc utiliser les valeurs UFL et PDI de l'Alimentation des ruminants (INRA, 1978).

\section{TABLEAU 1 a}

Valeur nutritive et composition chimique des aliments.

Nutritive value and chemical composition of food.

\begin{tabular}{|c|c|c|}
\hline \multirow[b]{2}{*}{$\begin{array}{l}\mathrm{UFL} / \mathrm{kg} \text { MS } \ldots \ldots \ldots \ldots \ldots \\
U F L / k g D M\end{array}$} & $\begin{array}{l}\text { Fourrage Pangola } \\
\text { Pangola grass }\end{array}$ & $\begin{array}{l}\text { Aliment concentré composé } \\
\text { Concentrate }\end{array}$ \\
\hline & 0,79 & 1,00 \\
\hline $\begin{array}{l}\text { MAD g/kg MS } \\
D C P \mathrm{~g} / \mathrm{kg} \mathrm{DM}\end{array}$ & 81 & 149 \\
\hline $\begin{array}{l}\text { PDIN g/kg MS } \ldots \ldots \ldots \ldots \ldots \\
P D I N \text { g/kg DM }\end{array}$ & 78 & 123 \\
\hline $\begin{array}{l}\text { PDIE g/kg MS } \ldots \ldots \ldots \ldots \ldots \\
P D I E \text { g } / \mathrm{kg} \mathrm{DM}\end{array}$ & 88 & 109 \\
\hline $\begin{array}{l}\text { Cellulose brute }(\mathrm{g} / \mathrm{kg} \mathrm{MS}) \ldots \ldots \ldots \\
\text { Crude Fibre }(\mathrm{g} / \mathrm{kg} \mathrm{DM})\end{array}$ & 332 & - \\
\hline $\begin{array}{l}\operatorname{MAT}(\mathrm{g} / \mathrm{kg} \mathrm{MS}) \\
C P(\mathrm{~g} / \mathrm{kg} D M)\end{array}$ & 120 & - \\
\hline $\begin{array}{l}\text { Matière minérale (g/kg MS) } \ldots \ldots \\
\text { Ash }(g / k g D M)\end{array}$ & 99 & - \\
\hline $\begin{array}{l}\text { Matière sèche }(\%) \quad \ldots \ldots \ldots \ldots \ldots \\
\text { Dry matter }(\%)\end{array}$ & 23 & 88 \\
\hline \multicolumn{3}{|c|}{$\begin{array}{l}\text { UFL : Unité fourragère lait : which is equivalent to the NEL content of one } \mathrm{kg} \text { standard barley (See " Alimentation } \\
\text { des ruminants }, I N R A, 1978 \text { ). }\end{array}$} \\
\hline \multicolumn{3}{|c|}{$\begin{array}{l}\text { DCP : Digestible Crude Protein = MAD : Matières Azotées Digestibles (MAD }=\text { MAT } \times \text { da). } \\
\text { PDIN : Protéine Digestible dans l'Instestin permise par l'azote fermentescible dans le rumen = Protein truly digestible } \\
\text { in the small intestine and allowed by its degradable nitrogen content. } \\
\text { PDIE : Proténe Digestible dans l'Intestin permise par l'énergie fermentescible dans le rumen = Protein truly } \\
\text { digestible in the small intestine and allowed by its degradable energy sources in the diet. }\end{array}$} \\
\hline
\end{tabular}

\section{A. Mesures climatiques}

\section{Paramètres macroclimatiques}

Ces paramètres sont relevés quotidiennement dans un poste météorologique standardisé situé à $100 \mathrm{~m}$ du lieu d'expérimentation. Il s'agit du rayonnement global $(\mathrm{Rg}=$ rayonnement solaire direct + rayonnement diffus $)$, de la pluviométrie, des températures maximales (Tmax) et minimale (Tmin) ainsi que de l'humidité relative maximale (HR max) et minimale (HR min). 


\section{Paramètres microclimatiques}

La température de la boule noire (TB), résultante des échanges thermiques radiatifs et convectifs, constitue un indice de caractérisation du microclimat (voir Missenard, 1931 ; Berbigier, 1982). Le thermomètre placé au niveau des animaux est lu entre 11 et $13 \mathrm{~h}$.

\section{B. Mesures thermophysiologiques et zootechniques}

La température rectale (TR) mesurée au moyen d'un thermomètre médical et le rythme respiratoire (RR) mesuré par comptage des battements du flanc pendant $1 \mathrm{mn}$, sont relevés entre $11 \mathrm{~h}$ et $13 \mathrm{~h}$ à 31 reprises regroupées en trois périodes : Période I (septembre-octobre 1982), Période II (novembre-décembre-janvier), Période III (maijuin 1983).

Les régimes sont appliqués à partir du $16 / 8$ et le premier contrôle alimentaire est effectué le 23/9, laissant ainsi une période d'adaptation pré-expérimentale d'un mois. Les pesées bihebdomadaires et les contrôles alimentaires durent jusqu'au 29/6/83. Les animaux de 4 blocs sur 6 sont alors abattus après un jeûne de $12 \mathrm{~h}$; le poids vif après jeûne, le poids vif vide, le poids de carcasse, le poids des différents organes et des viscères est enregistré. Les animaux des deux autres blocs sont conservés pour la reproduction.

On entendra par carcasse l'animal dépecé, décapité entre l'occipital et la $1^{\text {re }}$ vertèbre cervicale (atlas), éviscéré et dont les membres sont sectionnés au niveau des articulations carpe-métacarpe et tarse-métatarse. Cette présentation de carcasse diffère cependant de celle adoptée traditionnellement en Guadeloupe et dans d'autres pays des Caraïbes (Montserrat, Antigua) où l'on conserve la peau.

\section{Analyse statistique}

Nous appliquons un modèle d'analyse de variance à trois variables croisées (climat, alimentation, bloc ; SNEDECOR et Cochran, 1971). Seule, l'interaction climat $\times$ alimentation est prise en compte. Lorsqu'elle est significative, des tests $t$ entre les lots

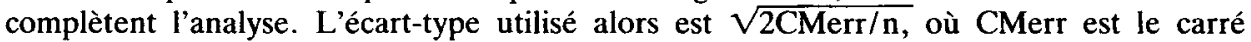
moyen résiduel de l'analyse de variance et $\mathrm{n}$ le nombre de répétitions (blocs). Lorsque les valeurs se présentent sous la forme d'un pourcentage (cas des abats), elles sont traitées au préalable par la transformation $x^{\prime}=\operatorname{Arc} \operatorname{Sin} \sqrt{x}$, afin d'en normaliser la distribution.

Cette analyse est appliquée aux moyennes relatives à la période expérimentale pour les résultats de consommation et de croissance. En ce qui concerne les données thermophysiologiques, qui varient de façon non négligeable avec le temps, des analyses ont été faites sur les moyennes de chacune de trois périodes définies plus haut. De plus, l'évolution au cours du temps a été testée au moyen des tests $t$ de paire effectués à l'intérieur de chaque lot (intra-lot), chaque pair étant constituée par les valeurs observées sur un même animal au cours de deux périodes différentes (inter-période).

Les animaux ayant été abattus à la même date et non pas à poids vif égal, nous avons effectué, pour les résultats d'abattage, une analyse de covariance sur le poids vif vide (PVV). 


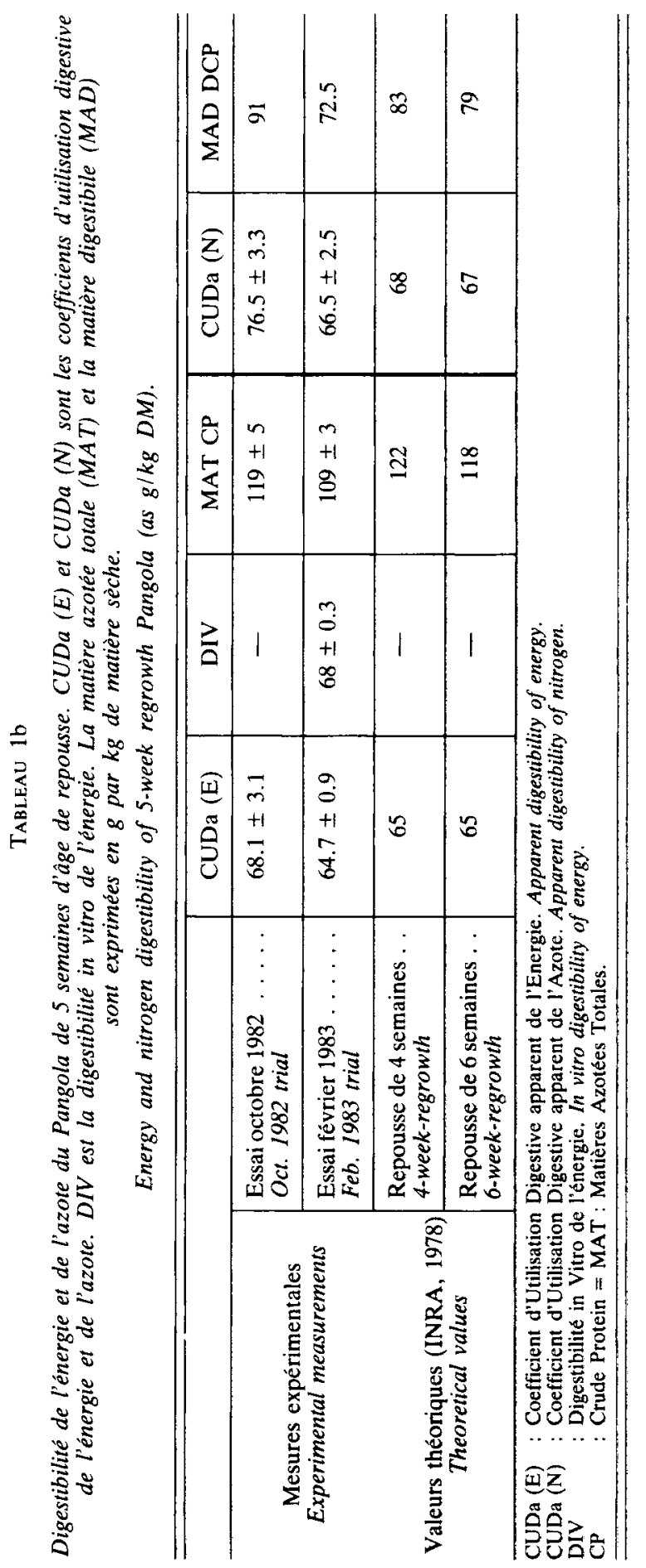




\section{Résultats}

\section{A. Variables climatiques}

Les conditions climatiques moyennes (moyennes \pm écart-type) de septembre 1982 à juin 1983 et la température boule moyenne sont rapportées dans le tableau 2. La pluviométrie est très irrégulière : les pluies se situent principalement en début d'expérience (décembre $=$ saison des pluies) et un peu à la fin (juin).

\section{B. Thermophysiologie}

Le tableau 3 donne les moyennes des températures rectales, des rythmes respiratoires ainsi que les caractéristiques du microclimat pour les trois périodes.

\section{Température rectale (TR) (tabl. 3)}

- La TR est significativement plus élevée chez les boucs exposés au soleil que chez ceux maintenus à l'ombre, uniquement en période III, période qui n'est pas la plus chaude.

- En période I, la plus chaude, la TR est plus élevée chez les boucs nourris avec le bas régime.

- Il n'y a pas d'interaction climat-alimentation sur TR.

TABLEAU 2

Conditions climatiques moyennes (moyenne \pm écart-type).

Climatic conditions (mean \pm standard deviation).

\begin{tabular}{|c|c|c|c|}
\hline $\begin{array}{l}\text { Nombre de mesures } \\
\text { Number of measurements }\end{array}$ & \multicolumn{2}{|c|}{$\begin{array}{l}\text { Paramètres climatiques } \\
\text { Climatic parameters }\end{array}$} & $\begin{array}{c}\text { Moyenne } \pm \text { Ecart-type } \\
\text { Mean } \pm \text { Standard deviation }\end{array}$ \\
\hline \multirow{5}{*}{$\mathrm{n}=300$} & \multicolumn{2}{|c|}{$\begin{array}{l}\mathrm{T} \max \left({ }^{\circ} \mathrm{C}\right) \ldots \ldots \ldots \ldots \\
\text { maximum temperature }\end{array}$} & $28.5 \pm 1.4$ \\
\hline & \multicolumn{2}{|c|}{$\begin{array}{l}\mathrm{T} \min \left({ }^{\circ} \mathrm{C}\right) \ldots \ldots \ldots \ldots \ldots \ldots \\
\text { minimum temperature }\end{array}$} & $23.1 \pm 1.9$ \\
\hline & \multicolumn{2}{|c|}{$\begin{array}{l}\mathrm{HR} \max (\%) \ldots \ldots \ldots \\
\text { maximum relative humidity }\end{array}$} & $\simeq 100$ \\
\hline & \multicolumn{2}{|c|}{$\begin{array}{l}\mathrm{HR} \min (\%) \ldots \ldots \\
\text { minimum relative humidity }\end{array}$} & $75 \pm 7$ \\
\hline & \multicolumn{2}{|c|}{$\begin{array}{l}\mathrm{Rg}\left(\mathrm{MJ} / \mathrm{cm}^{2} \times \text { jour }\right) \ldots \ldots \\
\text { global radiation }\left(M J / \mathrm{cm}^{2} \times \text { day }\right)\end{array}$} & $18.0 \pm 4.6$ \\
\hline \multirow{2}{*}{$\mathrm{n}=31$} & \multirow{2}{*}{$\begin{array}{l}\text { Température boule }\left({ }^{\circ} \mathrm{C}\right) \\
\text { Black globe temperature } \\
\left({ }^{\circ} \mathrm{C}\right)\end{array}$} & $\begin{array}{l}\text { Soleil } \\
\text { Sun }\end{array}$ & $37.7 \pm 5.0$ \\
\hline & & $\begin{array}{l}\text { Ombre } \\
\text { Shade }\end{array}$ & $30.2 \pm 2.0$ \\
\hline
\end{tabular}




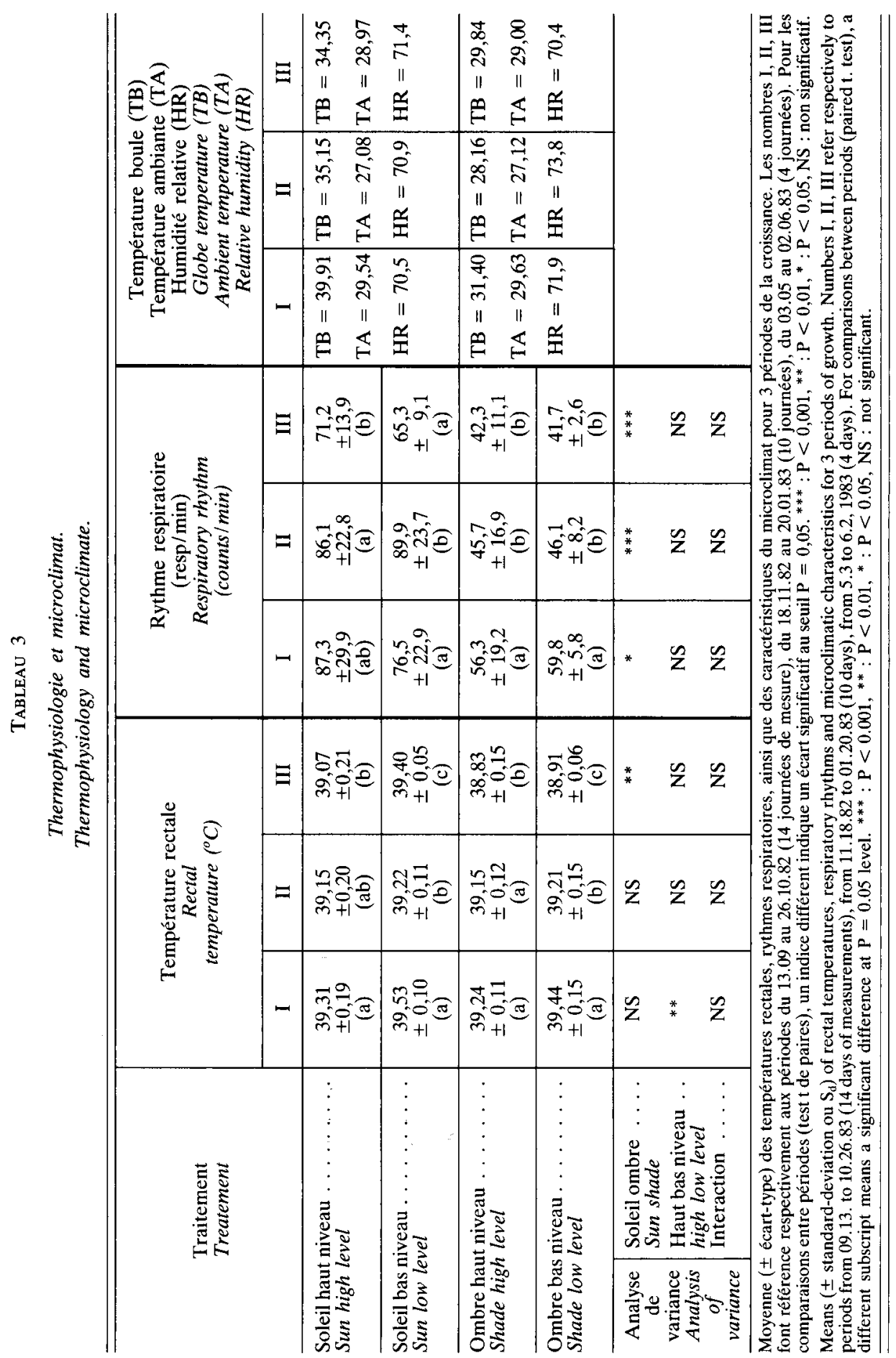




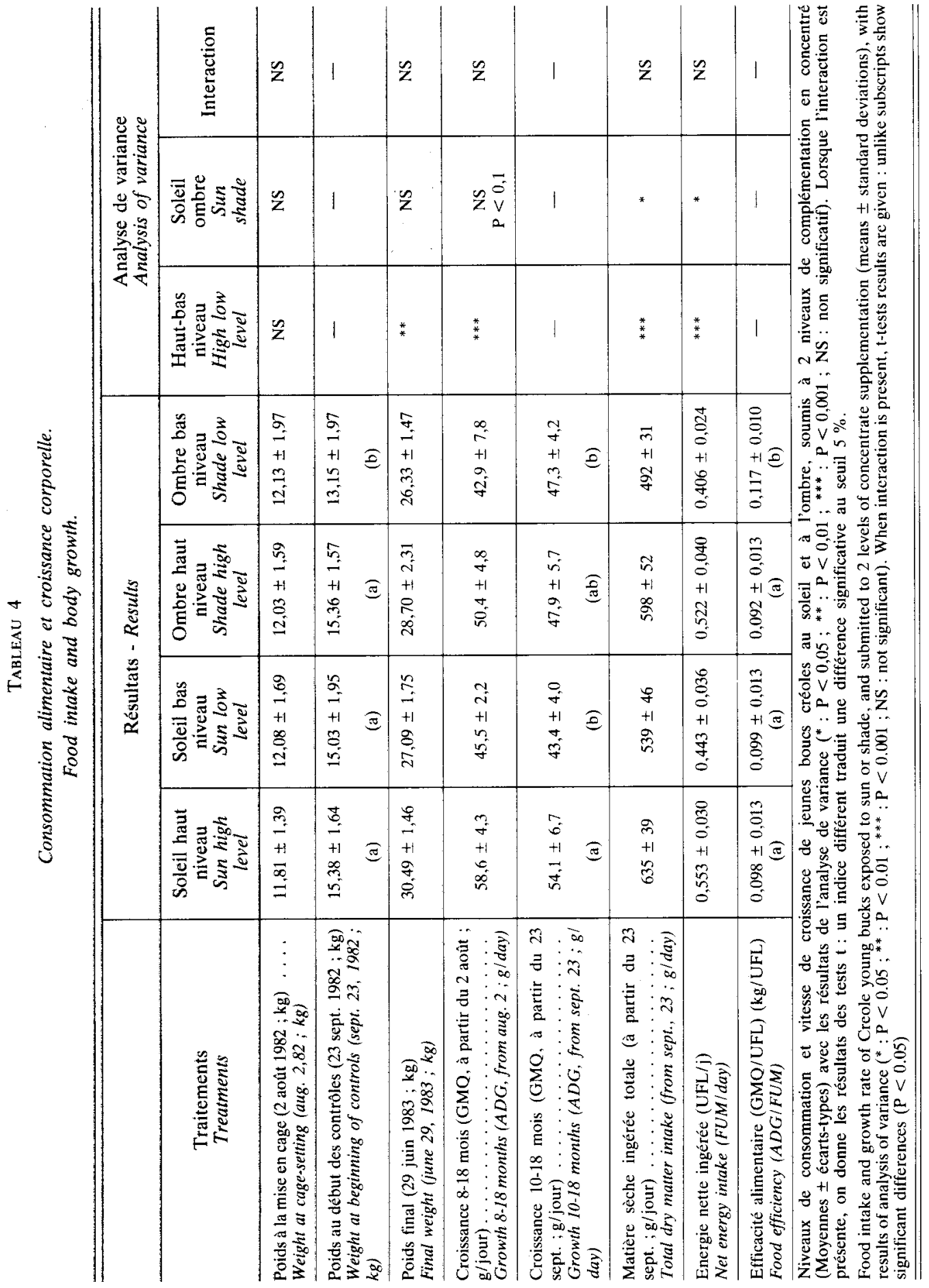


- Quel que soit le lot d'animaux, la TR décroit au cours des trois périodes, c'est-àdire en fonction du temps.

\section{Rythme respiratoire (RR) (tabl. 3)}

- Aux trois périodes, le RR est significativement plus élevé chez les boucs élevés au soleil, et cet effet s'accentue avec le temps malgré la chaleur moins intense.

- Par contre, il n'y pas d'effet du niveau alimentaire sur RR.

- Il n'y a pas d'interaction climat-alimentation sur RR.

- Le RR décroit au cours du temps (moins nettement pour le lot SB que pour les autres lots), aussi bien au soleil qu'à l'ombre, alors que TB et TA diminuent entre les périodes I et II, mais pas entre les périodes II et III.

\section{Vitesse de croissance (GMQ) et consommation alimentaire (MSI)}

(tabl. 4)

La courbe de vitesse de croissance présente un profil classique à trois pentes pour ce qui concerne les boucs des lots SH, SB et $\mathrm{OH}$ et anormal pour le lot OB. Celui-ci se caractérise par un très faible taux de croissance entre la mise en lots et le premier contrôle alimentaire ( 2 boucs ont maigri de respectivement 0,4 et $2,6 \mathrm{~kg}$ en 2 mois). Le calcul du GMQ à partir du premier contrôle alimentaire donne 47 g (GMQ 10-18 mois), soit une vitesse de croissance égale à celle du lot $\mathrm{OH}$ mais pour une quantité de MSI très significativement plus faible (100 g de moins par jour) : l'efficacité alimentaire apparente ( $\mathrm{kg}$ de gain/MSI ou $\mathrm{kg}$ de gain/énergie ingérée, AMES et BRINK, 1977), plus élevée que pour les autres lots, traduirait un phénomène analogue à une "croissance compensatrice ». Par contre, le calcul du GMQ dès la mise en lots donne une valeur de $42 \mathrm{~g}$ (GMQ 8-18 mois), soit une valeur nettement inférieure à celle du lot $\mathrm{OH}$. Un doute subsiste donc sur les conclusions relatives à ce lot.

La quantité de matière sèche ingérée varie au cours du temps : elle est minimale fin septembre (saison humide) et maximale fin février (saison sèche).

- L'effet de complémentation est toujours très significatif et se traduit, pour le même traitement climatique, par un poids final supérieur, une croissance plus rapide et des quantités ingérées supérieures pour le niveau de complémentation le plus haut ; par contre, l'efficacité alimentaire apparente n'est pas affectée.

- L'exposition au soleil semble être bénéfique aux animaux : le GMQ (à partir du 2 août) a tendance à être plus élevé bien que cet effet n'atteigne pas la signification à 5 p. $100(P<0,10)$ et les quantités ingérées sont significativement supérieures $(P<0,05)$. L'efficacité alimentaire apparente n'est pas non plus affectée. L'analyse de variance bihebdomadaire confirme que l'effet stimulant du soleil sur MSI commence le 9 février.

- Il n'y a pas d'interaction climat-alimentation sur les paramètres de croissance ou de consommation.

\section{Résultats d'abattage}

La covariable PVV n'a d'effet significatif $(P<0,05)$ que sur la compacité de carcasse et le pourcentage de gras du $5^{\mathrm{e}}$ quartier (gras interne). Dans tous les autres 


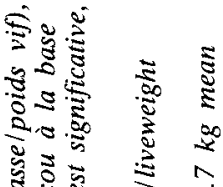

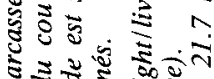

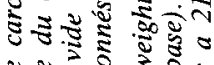
ช

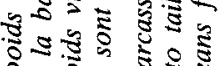

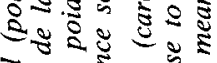
उ $20.5 \%$

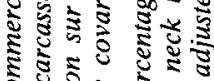

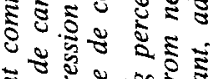
ริ ช इ ฐี

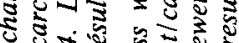
ชี

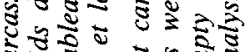
n ह จ จ 造 ६ ชี ₹ 昰范 उ

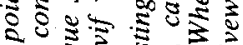

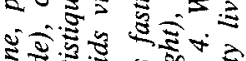

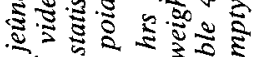

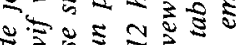
<ริ के के ๆ बें

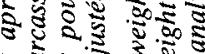

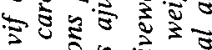
के

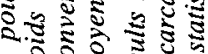

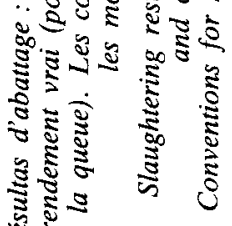

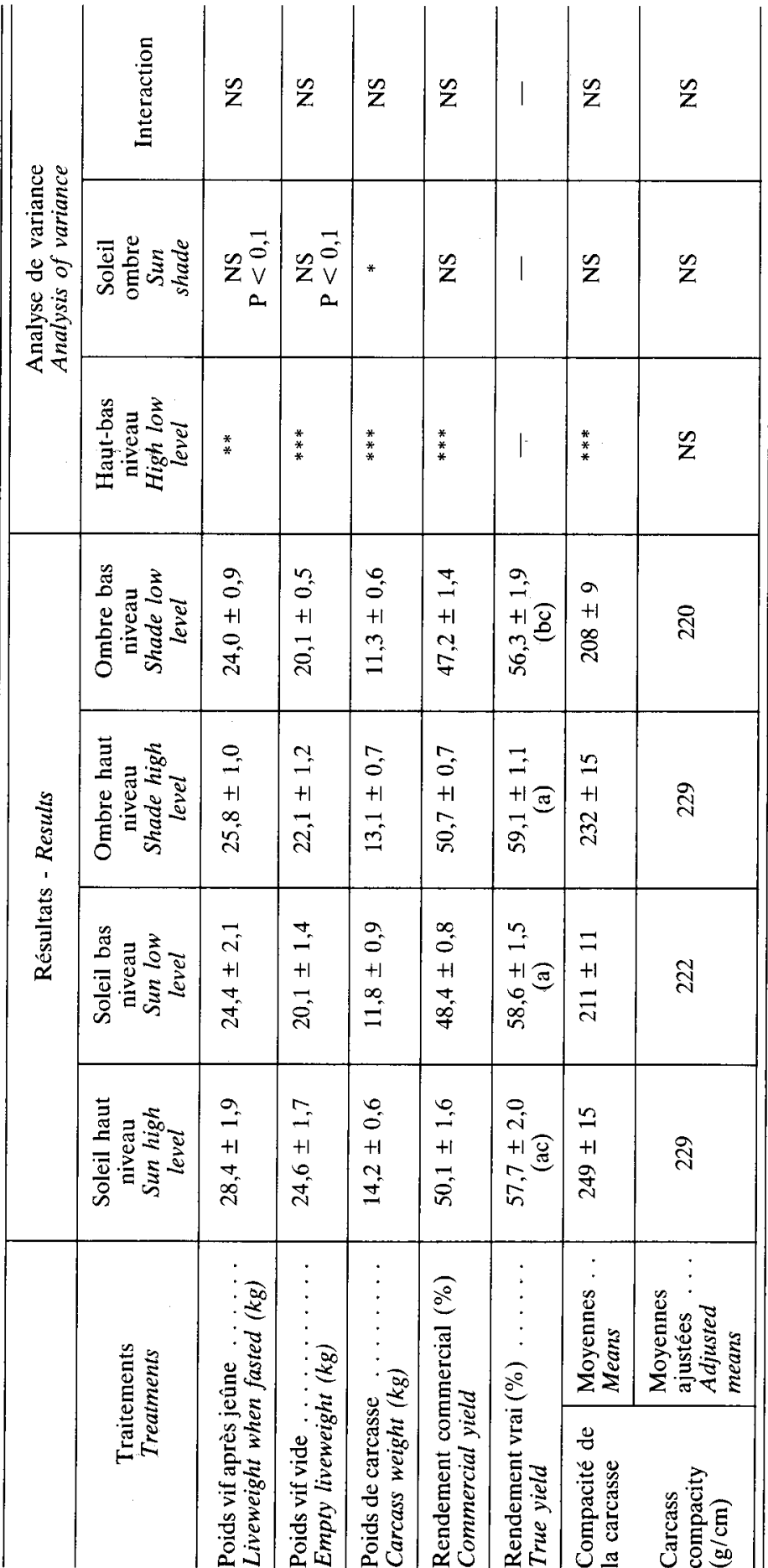


cas, son effet est extrêmement faible ; aussi, ne présentons nous les moyennes ajustées pour la covariable que dans le cas de ces deux paramètres (tabl. 5 et 6).

\section{Caractéristiques de carcasse (tabl. 5)}

Le haut niveau de complémentation conduit à un poids vif, un poids vif vide, un poids de carcasse ainsi qu'à un rendement commercial (poids de carcasse/poids vif) très significativement supérieurs par rapport au bas niveau. Il n'exerce pas d'effet direct sur la compacité de carcasse ajustée pour le PVV : les différences de compacité de carcasse observées sont intégralement prises en compte par le poids. Le rendement vrai (poids de carcasse/poids vif vide) ne semble pas affecté par le haut niveau de complémentation.

L'exposition au soleil tend à produire un poids vif et un poids vif vide $(\mathrm{P}<0,1)$ plus élevés. Cet effet est significatif $(\mathrm{P}<0,05)$ pour le poids de carcasse. Les autres paramètres ne sont pas affectés par l'exposition au soleil.

L'interaction climat-alimentation n'est présente que dans le cas du rendement vrai. Elle est surtout due à une valeur plus faible de ce paramètre pour le lot ombre-bas niveau et faiblement significative $(\mathrm{P}<0,05)$.

\section{Poids des abats (en $\%$ du poids vif vide, tabl. 6)}

Le pourcentage du poids vif vide représenté par les différents abats dépend peu des traitements, bien que certains effets isolés atteignent le seuil $\mathbf{P}<0,1$. Toutefois, le poids du tractus digestif vide est d'autant plus élevé que les animaux consomment plus d'herbe (bas niveau de complémentation, $\mathrm{P}<0,05$ ), et le poids du foie augmente de façon très significative chez les animaux exposés au soleil $(P<0,001)$. Le gras du $5^{\mathrm{c}}$ quartier est significativement supérieur chez les boucs maintenus à l'ombre $(P<0,05)$.

\section{Discussion}

\section{A. Thermophysiologie}

L'exposition au soleil s'accompagne toujours d'une augmentation du rythme respiratoire, contribuant ainsi à une évaporation de chaleur latente plus importante que chez les animaux à l'ombre (BoRUt et al., 1979) ; la température rectale des boucs au soleil est légèrement plus élevée que celle des boucs à l'ombre. Ainsi, contrairement à ce que l'on pouvait attendre, les boucs exposés à long terme au soleil ne sont pas en état de "stress thermique " mais présentent seulement les signes d'une thermorégulation active et efficace (Sergent, 1985 ; Sergent et al., 1985, Sergent, Berbigier, Ravault, non publiées). Par rapport au diagramme de Mount (1974) les boucs créoles se situent souvent hors de leur zone de moindre effort thermorégulatoire puisqu'il y a augmentation marquée des pertes évaporatives respiratoires : dans nos conditions expérimentales, quasi-naturelles, la température critrique supérieure (TCS) devrait être comprise entre $27^{\circ} \mathrm{C}$ et $32{ }^{\circ} \mathrm{C}$ (correspondant environ à $34^{\circ} \mathrm{C}<\mathrm{TB}<42^{\circ} \mathrm{C}$ ) et serait comparable à celle rapportée pour le caprin nain d'Afrique de l'Ouest, $25^{\circ} \mathrm{C}<\mathrm{TCS}<35^{\circ} \mathrm{C}$, en chambre respiratoire (LuiTiNG et al., 1985). La TR plus élevée chez les boucs du régime B en cas de chaleur intense, peut s'expliquer par l'extra-chaleur de digestion plus 


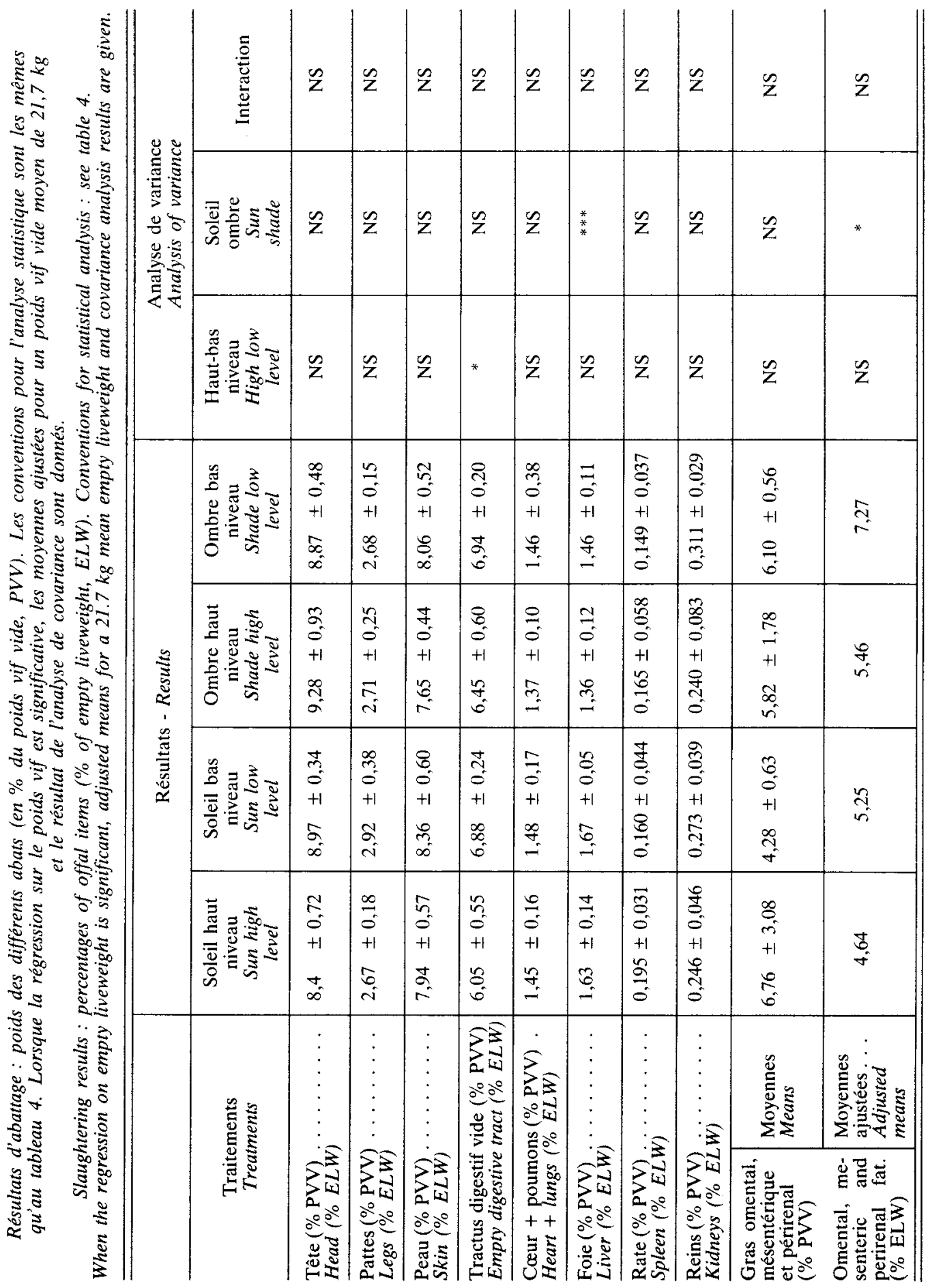


importante puisque la proportion de fibres cellulosiques dans cette ration est plus élevée (Vermorel, 1978). Indépendamment de la température extérieure, la TR diminue au cours des trois périodes : la thermosensibilité des boucs diminue légèrement en fonction de l'âge (TwaITEs, 1967).

\section{B. Croissance, consommation alimentaire}

Les valeurs du gain de poids obtenues entre 8 et 18 mois $(55 \mathrm{~g} / \mathrm{j}$ pour le régime $\mathbf{H}$ et $44 \mathrm{~g} / \mathrm{j}$ pour le régime $B$ ) semblent assez caractéristiques de la vitesse de croissance des jeunes boucs créoles. Le besoin azoté des animaux est couvert puisque d'après XANDE (1985, non publié) une quantité de $22 \mathrm{~g}$ de PDI permet $100 \mathrm{~g}$ de GMQ, le régime $B$ autorisant alors un gain de poids de $75 \mathrm{~g} / \mathrm{j}$. Le facteur nutritif limitant de la croissance serait donc l'énergie de la ration. Une meilleure vitesse de croissance $(75 \mathrm{~g} /$ j) entre 3,5 et 8 mois d'âge est rapportée par XANDE (in ChEMINEAU et al., 1984) avec des boucs recevant une ration mixte enrichie en tourteau de soja et élevés en stabulation libre couverte en Basse-Terre (climat moins chaud).

Il est probable qu'avec le niveau B, la capacité d'ingestion de fourrage soit saturée alors que l'apport plus élevé de concentré dans la ration $\mathrm{H}$ permet d'augmenter la quantité ingérée totale, en réduisant le temps de transit et l'encombrement des aliments. Cette augmentation entraîne une meilleure vitesse de croissance, sans que l'efficacité alimentaire exprimée ici par le rapport du gain de poids sur la valeur énergétique de la ration ingérée, ne soit affectée.

Un résultat inattendu est l'effet bénéfique de l'absence d'ombrage, effet qui atteint le niveau de signification à $5 \%$ dans le cas des quantités ingérées : il est faible par rapport à l'effet du haut niveau de complémentation, mais il tend à prouver que les boucs créoles sont thermotolérants et peuvent maintenir une production dans les conditions les plus chaudes que l'on puisse trouver en Guadeloupe (zone sèche, pas d'abri, ventilation coupée par les contrevents), contrairement aux bovins Zébus créoles, chez lesquels, dans les mêmes conditions, on observe une diminution des quantités ingérées (Berbigier, 1983). Cette capacité d'ingestion supériewre chez les boucs exposés au soleil pourrait être en relation avec les taux élevés de prolactine plasmatique, hormone pour laquelle est évoqué un rôle dans la modification des processus digestifs en réponse à un changement de l'environnement thermique (Christopherson, 1985).

Le déficit initial de croissance des animaux du lot $O B$ ne semble affecter de façon nette que leur croissance ultérieure. L'efficacité alimentaire du lot $\mathrm{OB}$, supérieure à celle des trois autres lots, pourrait s'expliquer par une différence de composition du gain corporel (développement plus important du tube digestif, Morand-FeHR, 1981). DEVENDRA (1981) rapporte comme taux de conversion alimentaire (énergie ingérée $/ \mathrm{kg}$ de gain, FEHr et al., 1976) une valeur de 21,5 à $26 \mathrm{Mcal}_{\mathrm{EM}} \mathrm{EM}^{*} / \mathrm{kg}$ gain de poids pour un caprin pesant entre 20 et $30 \mathrm{~kg}$ de poids vif. Dans le même système d'élevage, les boucs créoles utilisent pour les niveaux de complémentation $\mathrm{H}$ et $\mathrm{B}$, respectivement 28,4 et $28,1 \mathrm{Mcal} \mathrm{EM} / \mathrm{kg}$ gain. Ceci correspond à une efficacité alimentaire faible $(0,092$ à $0,117 \mathrm{~kg}$ gain/UFL) par comparaison à celle de taurillons créoles $(0,145 \mathrm{~kg}$ gain/UFV) ou des croisés Limousins-créoles $(0,162 \mathrm{~kg}$ gain/UFV) âgés de 10 à 15 mois (BERBIGIER \& SoPHiE, 1986). L'efficacité alimentaire des boucs créoles est également faible, comparée à celle des croisés Alpins-créoles, nourris avec un régime $\mathrm{H}: 0,108$ $\mathrm{kg} /$ UFL vs $0,145 \mathrm{~kg} / \mathrm{UFL}, \mathrm{P}<0,01$ (SERGENT, Berbigier, Sophie, donnees non publiées), ce qui suggère que les créoles utilisent moins bien le concentré.

(*) $\mathrm{EM}=$ énergie métabolisable. 


\section{Performances d'abattage}

La découpe de carcasse adoptée dans ce travail par l'abattoir expérimental de la Station de Zootechnie du Centre INRA de Guadeloupe correspond donc à la découpe standard récemment précisée par Colomer-Rocher, Morand-Fehr \& Kirton (1987). Le rendement commercial de carcasse est meilleur pour le haut niveau de complémentation, en raison du poids du contenu digestif relatif au poids vif qui est plus faible que pour les animaux de bas niveau (13,8 p. 100 contre 16,9 p. 100). Le poids relatif des autres abats ne semble pas dépendre du niveau de complémentation. Le rendement de carcasse observé (moyenne générale : 49 p. 100) est conforme à celui rapporté par Chemineau et al. (1984) sur la même race $(50,3$ p. 100). Le rendement commercial à l'abattage présentant généralement une faible variation, il n'est pas très étonnant qu'il se situe dans la plage $46-53$ p. 100 , variabilité de rendement probablement due aux différences dans les conditions expérimentales (alimentation et système de conduite, poids et âge à l'abattage, sexe...). Ce rendement est comparable à ceux de la race Kambing-Katjang de Malaisie (51 p. 100, Devendra, 1979), de la race Criollo du Venezuela en milieu plus aride (49 p. 100, Acosta, 1979, cité par Devendra \& Owen, 1983), de la chèvre rousse : (taille moyenne à petite) : 48 p. 100 (Sokoto du Nigéria, Alaku \& MorupPa, 1983 ; et Maradi du Niger, Robinet, 1967), de la race Fidji (taille moyenne, 46 p. 100, Hussain et al., 1983), de la chèvre du sud Tchad (taille petite à naine, 53 \%, Dumas, 1977) ainsi qu'aux rendements de races à viande "Rustiques » (52 p. 100, "Spanish goats» des U.S.A., Smiтh et al., 1982). Ces pourcentages de rendement en carcasse s'observent aussi chez les races "intensives " (Alpine française, 52 p. 100, Fehr et al., 1976 ; Saanen, Toggenburg, 47 à 52 p. 100, LAdipo, 1973). Enfin, il pourrait être intéressant d'étudier l'aptitude à l'engraissement chez le jeune bouc créole castré à un âge optimal restant à déterminer : le rendement de carcasse serait-il supérieur comme chez le Criollo $(+2,4$ p. 100, Acosta, 1979, cité par DevenDRA \& OWEN, 1983) ou chez la chèvre de Maradi (+ 5 p. 100, RoBinet, 1967) et ce type de production serait-il rentable, compte-tenu de la réduction probable de la vitesse de croissance?

L'augmentation très significative, au soleil, du poids du foie, peut être en relation avec le rôle de cette glande dans la thermorégulation. Cela correspondrait à une adaptation anatomo-physiologique comparable à l'accroissement (hypertrophie et hyperplasie) du muscle cardiaque de pores élevés sous les tropiques (AlAKU et al., 1984). La signification de ce phénomène reste à élucider, mais on pourrait s'orienter vers une augmentation ou une réorientation du métabolisme lipidique (lipolyse, MC ATEE \& TrenKLE, 1971) et du métabolisme protéique (anabolisme protéique) en liaison avec un taux élevé de prolactine plasmatique (SERGENT, 1985).

Par contre, le poids d'autres glandes endocrines (surrénales, thyroïde) est plus élevé à l'ombre qu'au soleil (Sergent, Berbigier, Sophie, données non publiées), résultat s'accordant alors avec les observations de RAy et al. (1968) \& STRAuB et al. (1976) indiquant que les hautes températures, chez le rat et le verrat, dépriment le poids des organes internes.

En conclusion, les boucs créoles se caractérisent par d'assez bonnes performances de croissance et d'abattage, compte tenu de leur format nain et de l'absence, jusqu'à présent, de sélection génétique. Ils présentent l'avantage d'être bien "adaptés " au plein air tropical, à tel point que celui-ci stimule leur capacité d'ingestion et semble améliorer légèrement $(\mathrm{P}<0,1)$ leur vitesse de croissance. Toutefois, cet effet "positif » du soleil sur la croissance est mis en évidence par comparaison avec des boucs 
« témoins » maintenus à l'ombre, situation climatique de référence qui peut avoir été «négative» sur l'état psycho-physiologique des animaux. La situation ombragée, semiconfinée, fait en sorte que les animaux à l'ombre, moins stimulés par l'environnement (au sens large), sont plus émotifs comme le montre leur comportement vis-à-vis de l'homme et l'évolution de leur cortisolémie au cours de la journée (SERGENT, 1985). Il est alors possible que leur consommation d'aliment, leur digestion et par conséquent leur croissance soient affectées.

Reçu en mars 1985.

Accepté en mars 1987.

\section{Remerciements}

Les auteurs expriment leur gratitude à la Station de Zootechnie du Centre INRA des Antilles, et en particulier à $D$. VINCENT et P. Marival de l'abattoir, pour leur aide efficace, ainsi qu'à Michelle Huc, Marie-Laure CLEDOR, Roselyne VANSUYT et Nicolle Neybecker pour la dactylographie.

\section{Summary}

Influence of humid tropical climate and level of feeding on thermoregulation and growth and slaughtering performances of young male Creole goats in Guadeloupe.

Twenty-four $246 \pm 45$ day-old young male Creole Dwarf goats of Guadeloupe (French West Indies), weighing $12.0 \pm 1.5 \mathrm{~kg}$ at cage setting were submitted to two climatic conditions (sun $\mathrm{S}$ or no sun NS, Table 2) and two levels of feeding (high $\mathbf{H}$ or low $\mathrm{L}$ concentrate level), according to a $2 \times 2$ factorial design. The former were sun exposed while the second were sheltered. All bucks were daily fed ad libitum Pangola grass and in addition $\mathrm{H}$ bucks ones were given $270 \mathrm{~g} / \mathrm{d}$ dry matter (DM) or concentrate, $\mathrm{L}$ ones $90 \mathrm{~g}$. The experiment was conducted for 10 months.

Rectal temperature of $\mathrm{L}$ bucks was higher than that of $\mathrm{H}$ ones only when the ambient temperature was particularly hot and respiratory rhythm was independent of the level of feeding (Table 3). In $\mathrm{H}$ bucks, food intake $(617 \mathrm{~g} \mathrm{DM} / \mathrm{d}$ vs $516 \mathrm{~g} \mathrm{DM} / \mathrm{d})$, average daily gain $(54.5 \mathrm{~g} \mathrm{vs}$ $44.2 \mathrm{~g})$, empty live weight $(23.4 \mathrm{~kg} v s 20.1 \mathrm{~kg})$ and carcass weight $(13.7 \mathrm{~kg}$ vs $11.6 \mathrm{~kg})$ were significantly improved $(P<0.001)$ (Tables 4 and 5). The true carcass yield was not affected, nor the omental, mesenteric and perirenal fat (Tables 5 and 6 ).

$\mathrm{S}$ bucks exhibited a higher food intake (total DMI : $587 \mathrm{~g} / \mathrm{d} v s 545 \mathrm{~g} / \mathrm{d}$ for NS, $\mathrm{P}<0.05$ ), carcass weight $(13.0 \mathrm{~kg}$ vs $12.2 \mathrm{~kg}, \mathrm{P}<0.05)$, liver weight $(1.65 \%$ of empty live weight $v S$ $1.41 \%, \mathrm{P}<0.001)$, and a lower omental, mesenteric and perirenal fat percentage $(4.9 \% v s$ $6.4 \%, \mathrm{P}<0.05$ ) (Tables 4,5 and 6 ).

In the hottest and quasi-natural conditions prevailing in Guadeloupe, Creole Dwarf bucks were heat-tolerant and integral open air breeding, with sufficient forage availability and well-being, was not a limiting factor since performance were satisfactory.

Key words: Wet tropics, French West Indies, young male creole Dwarf goat, thermal regulation, meat production. 


\section{Références bibliographiques}

Acosta I.H., 1979. Peso vivo, peso en canal y rendimiento en canal de ovinos y caprinos sacrificados en el frigorifico industrial. Bolivar, C.A. del Estado Zulia, Universitad de Zulia, Maracaibo, Venezuela.

Alaku O., Moruppa S.M., 1983. Dry season weight losses in Red Sokoto goats reared in the Sahel region of northeastern Nigeria. Int. J. Biometeor., 27, 143-156.

Alaku O., Steinbach J., Avery P.J., 1984. Effect of season of birth and sex on heart weight and body weight and their interrelationship in pigs reared in the tropics. Anim. Prod., 38, 495502 .

Alexandre G., 1983. Production laitière des chèvres Créoles : facteurs de variation, influence sur la croissance des jeunes. Thèse E.N.S.A. et Université de Rennes I, 138 pages.

Ames D.R., Brink D.R., 1977. Effect of temperature on lamb performance and protein efficiency ratio. J. Anim. Sci., 44, 136-140.

Berbigier P., 1982. Analyse physique des pertes thermiques de l'animal vers son environnement. Influence des facteurs climatiques. In : Actions du climat sur l'animal au pâturage, INRA, Services des Publications, Route de Saint-Cyr, Versailles, p. 65-96.

Berbigier P., 1983. Effet du climat tropical humide sur la consommation d'aliment et d'eau et sur la vitesse de croissance de taurillons Créoles en Guadeloupe. Ann. Zootech., 32, 93-108.

Berbigier P., Sophie S.A., 1986. Performances de croissance et d'abattage de taurillons Limousin$\times$ Créoles et Créoles élevés au soleil et à l'ombre en Guadeloupe (Antilles Françaises). Rev. Elev. Med. Vet. Pays trop., 39 (1), 81-89.

Borut A., Dmiel R., Shкolnik A., 1979. Heat balance of resting and walking goats : comparison of climatic chamber and exposure in the desert. Physiol. Zool., 52, 105-112.

Chemineau P., Cognie Y., Xande A., Peroux F., Alexandre G., Levy F., Shitalou E., Beche J.M., Sergent D., Camus E., Barre N., Thimonier J., 1984. Le cabri Créole et ses caractéristiques zootechniques. Monographie. Rev. Elev. Med. Vet. Pays trop., 37, 225-228.

Christopherson R.J., 1985. The thermal environment and the ruminant digestive system. In : Stress physiology in livestock, M.K. Yousef éd., C.R.C. Press, Boca Raton (Flor. U.S.A.), 163-180.

Colomer-Rocher F., Morand-Fehr P., Kirton A.H., 1987. Standard methods and procedures for goat carcass evaluation and jointing. Livestock Prod. Sci., 16 (2) (sous presse).

Devendra C., 1979. Potentiel de production de caprins et d'ovins dans la région de l'A.S.E.A.N. Rev. Mond. Zootech., 32, 33-41.

Devendra C., 1981. The goat in the humid tropics. In : Goat production, C. Gall éd., Acad. Press, London (G.B.), 557-572.

Devendra C., Owen J.E., 1983. Aspects quantitatifs et qualitatifs de la production de viande caprine. Rev. Mond. Zootech., 47, 19-29.

Dumas R., 1977. Etude sur l'élevage des petits ruminants du Tchad. Données sur les rendements en carcasse à l'abattoir. Maisons-Alfort, I.E.M.V.T., document interne.

Fehr P.M., Sauvant D., Delage J., Dumont B.L., Roy G., 1976. Effect of feeding methods and age at slaughter on growth performances and carcass characteristics of entire young male goats. Livest. Prod. Sci, 3, 183-194.

Hussain M.Z., Naidu R., Tuvuki I., Singh R., 1983. Production et développement de l'élevage caprin à Fidji. Rev. Mond. Zootech., 48, 25-32.

INRA, 1978. Alimentation des ruminants. INRA éd., Services des Publications, Route de SaintCyr, 78000 Versailles, 621 pages.

LADIPO J.K., 1973. Body composition of male goats and characterization of their fat depots. Ithaca, Cornell University, Thèse, phD.

Luiting P., Montsma G., Verstegen M.W.A., Hofs P., Van Der Hel W., Zujlker J.W., 1985. Effects of high ambient temperatures on the metabolism of West African dwarf goats. I. Int. J. Biometeor., 29, 11-22. 
Mc Atee J.W., Trenkle A., 1971. Effect of feeding, fasting, glucose or arginine on plasma prolactin levels in the bovine. Endocrinology, 89, 730-734.

Missenard A., 1931. In : Missenard, 1966. Cours supérieur de chauffage, ventilation et conditionnement d'air. Eyrolles éd. Paris.

Morand-Fehr P., 1981. Growth. In : Goat production. C. Gall ed., Acad. Press, London (G.B.), 253-281.

Mount L.E., 1974. Thermal neutrality. In : Heat loss from animals and man : assessment and control. 425-439 Monteith J.L. and Mount L.E. ed., Butterworths, London (G.B.).

RaY D.E., RoubiceK C.B., HANidi M., 1968. Organ and gland weights of rats chronologically exposed to $22^{\circ}$ and $35^{\circ} \mathrm{C}$. Growth, 32, 1-2.

Robinet A.H., 1967. La chèvre rousse de Maradi. Son exploitation et sa place dans l'économie et l'élevage de la République du Niger. Rev. Elev. Med. Vét. Pays trop., 20, 129-196.

SERGENT D., 1985. Régulations endocriniennes et adaptation physiologique au climat tropical humide chez le bouc Créole: éléments suggérant un rôle de la prolactine dans la thermorégulation. Thèse de Doc. $3^{\text {e }}$ cycl, Univ. P. \& M. Curie, Paris VI, 138 pages.

Sergent D., Berbigier P., Kann G., Fevre J., 1985. The effect of sudden solar exposure on thermophysiological parameters and on plasma prolactin and cortisol concentrations in male Creole bucks. Reprod. Nutr. Develop., 25, 629-640.

Sergent D., Berbigier P., Ravault J.P., 1987. Effect of prolactin inhibition on thermophysiological parameters, water and feed intake of sun-exposed male Creole goats in Guadeloupe (French West Indies). (Accepté pour publication dans J. Therm. Biol.).

Smith G.C., Riley R.R., Savell J.W., Shelton M., 1982. Yields of carcass and dress-off items and carcass quality-quantity measures for Angora and Spanish goats. In : "Proc. $3^{\text {rd }}$ Int. Conf. on goat production and disease ", 10-15 January, Tucson (Ariz. U.S.A.), 469-471.

Snedecor G.W., Cochran W.G., 1971. Méthodes statistiques. Association de Coordination Technique Agricole éd., 649 pages.

Straub G., Weniger J.N., Tawfic E.S., Steinhauf D., 1976. The effect of high environmental temperatures on fattening performance and growth of boars. Livestock Prod. Sci., 3, 65-74.

Twaites C.J., 1967. Age and heat tolerance in sheep. Int. J. Biometeor., 11, 209-212.

Vermorel M., 1978. Utilisation énergétique des produits terminaux de la digestion. In : Alimentation des ruminants. INRA éd., Service des Publications, Route de Saint-Cyr, 78000 Versailles, p. $47-88$. 